PROCEEDINGS OF THE

AMERICAN MATHEMATICAL SOCIETY

Volume 130, Number 9, Pages 2661-2667

S 0002-9939(02)06513-9

Article electronically published on March 13, 2002

\title{
COMPACTLY BOUNDED CONVOLUTIONS OF MEASURES
}

\author{
ADAM W. PARR \\ (Communicated by Andreas Seeger)
}

\begin{abstract}
In this paper we extend classical results concerning generalized convolution structures on measure spaces. Given a locally compact Hausdorff space $X$, we show that a compactly bounded convolution of point masses that is continuous in the topology of weak convergence with respect to $C_{c}(X)$ can be extended to a general convolution of measures which is separately continuous in the topology of weak convergence with respect to $C_{b}(X)$.
\end{abstract}

\section{INTRODUCTION}

The study of generalized convolution systems has been of interest to mathematicians for many years. Of particular interest in the last half of the twentieth century has been those situations where some structure placed upon a locally compact (Hausdorff) space $X$ will yield a continuous convolution on $M_{b}(X)$, the space of bounded Radon measures on $X$. To be more exact, when a continuous map $w: X \times X \rightarrow M_{b}(X)$ can be extended to a continuous map (a convolution) from $M_{b}(X) \times M_{b}(X) \rightarrow M_{b}(X)$, where $M_{b}(X)$ is endowed with the $C_{b}$-weak $\left(\tau_{b}\right)$ topology of weak convergence with respect to $C_{b}(X)$, the space of continuous bounded functions on $X$. This situation arises, for example, when $X$ is a group (see [2]), a hypergroup (see [3]), or a signed hypergroup (see this paper). In the group case the image of the original map $w$ consists entirely of point-mass (or Dirac) measures. For the hypergroup case, where the image of the map $w$ consists of probability measures, Jewett [3] used the "cone" topology which is equivalent to the $C_{b}$-weak topology on the subspace $M_{b}^{+}(X)$ consisting of positive measures.

In this paper we will show that if the map $w$ is "compactly bounded", and bounded by the standard norm on $M_{b}(X)$, then it can still be extended continuously to $M_{b}(X) \times M_{b}(X)$ even though its image may contain signed measures. Moreover, in order to have the extension be separately continuous with respect to the $C_{b}$-weak topology, it will only be necessary to require that the map $w$ is continuous with respect to the $C_{c^{-}}$weak $\left(\tau_{c}\right)$ topology.

\section{The Strict topology on $C_{b}(X)$}

An important tool in this paper will be the strict $(\beta)$ topology on $C_{b}(X)$, which lies between the compact-open $(\kappa)$ and the sup-norm $(\sigma)$ topologies. This topology is interesting and useful because, as was shown by C. Buck in the 1950 's, $M_{b}(X)$ is

Received by the editors April 6, 2001.

2000 Mathematics Subject Classification. Primary 43A99.

Key words and phrases. Signed hypergroup, convolution, strict topology.

(C)2002 American Mathematical Society 
the strict dual of $C_{b}(X)$ even if $X$ is not compact. In other words, $M_{b}(X)$ consists exactly of those linear functionals on $C_{b}(X)$ which are continuous with respect to the strict topology. For details concerning the strict topology we refer the reader to [1].

We begin by defining the strict topology and extending some basic results concerning the strict and $C_{b}$-weak topologies. Please note that throughout this paper all topological spaces are assumed to be locally compact and Hausdorff, and that we use the notation $C_{0}(X)$ to represent the space of continuous functions on $X$ which vanish at infinity.

Definition. A net $\left(f_{\alpha}\right)$ in $C_{b}(X)$ converges to $f \in C_{b}(X)$ with respect to the strict topology on $C_{b}(X)$ if for each $\phi$ in $C_{0}(X)$, the net $\left(\phi f_{\alpha}\right)$ converges to $\phi f$ uniformly.

Lemma 2.1. If $\left(f_{\alpha}\right)$ is a norm-bounded net in $C_{b}(X)$ which converges to $f$ in $C_{b}(X)$ with respect to the compact-open topology, then $\left(f_{\alpha}\right)$ converges to $f$ with respect to the strict topology.

Proof. Let $A=\left\{g \in C_{b}(X):\|g\| \leq \sup _{\alpha}\left\{\left\|f_{\alpha}\right\|,\|f\|\right\}<\infty\right\}$. Then $A$ is a norm bounded subset of $C_{b}(X)$. Hence, by Theorem 1(iv, v) in [1, the compact-open topology agrees with the strict topology on $A$. Thus $\left(f_{\alpha}\right)$ converges to $f$ with respect to the strict topology.

Lemma 2.2. The set, $F M_{b}(X)$, of measures on $X$ with finite support is $C_{b}$-weakly dense in $M_{b}(X)$.

Proof. The space $C_{b}(X)$ is a separating vector space of linear functionals on $M_{b}(X)$. Thus, by Theorem 3.10 in [5], $\left(M_{b}(X), \tau_{b}\right)$ is a locally convex space whose dual is $C_{b}(X)$. Let $S$ be the $\tau_{b}$-closure of $F M_{b}(X)$ in $M_{b}(X)$. By Theorem 1.13(c) in [5], $S$ is a closed linear subspace of $M_{b}(X)$. Suppose that $S \neq M_{b}(X)$ and let $\mu_{o} \in M_{b}(X) \backslash S$. By Theorem 3.5 in [5], there exists an $f \in C_{b}(X)$ such that $f\left(\mu_{o}\right)=1$ and $f(\mu)=0 \forall \mu \in F M_{b}(X)$. But this implies that for every $x \in X$ we have $f(x)=\delta_{x}(f)=f\left(\delta_{x}\right)=0$ which implies $f \equiv 0$ and hence $f\left(\mu_{o}\right)=0$ which is a contradiction.

\section{Convolution extension theorem}

The motivation for this paper is the study of signed hypergroups, and we note here that the hypotheses of the following lemma and theorem are satisfied by the axioms of a signed hypergroup which have appeared in [11. Note that the only restrictions on the map $w$, besides continuity, are that $w$ must be bounded in two ways; with respect to the standard norm on $M_{b}(X)$ and also with respect to compact sets.

Definition. Let $X$ and $Y$ be locally compact Hausdorff spaces. A map $w: X \rightarrow$ $M_{b}(Y)$ is said to be compactly bounded if for every compact subset $K \subseteq X$, the set $\bigcup\left\{\operatorname{supp}\left(w_{x}\right): x \in K\right\}$ is relatively compact in $Y$.

As the next theorem shows, one of the main benefits of compactly bounded maps is that $C_{b}$-weak continuity will be equivalent to $C_{c}$-weak continuity.

Theorem 3.1. Let $w: X \rightarrow M_{b}(Y)$ be compactly bounded and $C_{c}$-weakly continuous. Then $w$ is also $C_{b}$-weakly continuous. 
Proof. Let $f \in C_{b}(Y)$ and let $\left(x_{\alpha}\right)$ be a net in $X$ which converges to $x \in X$. We must show that the net $w_{x_{\alpha}}(f)$ converges to $w_{x}(f)$.

Choose a relatively compact open subset $V$ of $X$ with $x \in V$. Since $w$ is compactly bounded, the set $A=\bigcup_{x \in \bar{V}} \operatorname{supp}(w(x))$ is a relatively compact subset of $Y$. Choose a relatively compact open subset $W$ of $Y$ with $\bar{A} \subseteq W$ (where $\bar{A}$ is the closure of $A$ ). Since $Y$ is locally compact, we can choose a positive function $g \in C_{c}(Y)$ such that $g \equiv 1$ on $\bar{A}$ and $g \equiv 0$ on $Y \backslash W$. Since $w$ is $C_{c^{-}}$weakly continuous and $g f \in C_{c}(X)$, the net $w_{x_{\alpha}}(g f)$ converges to $w_{x}(g f)$.

Now, let $\epsilon>0$ be given. Choose an index $\alpha_{o}$ such that, for all $\alpha \geq \alpha_{o}, x_{\alpha} \in V$ and $\left|w_{x_{\alpha}}(g f)-w_{x}(g f)\right|<\epsilon$. Let $\alpha \geq \alpha_{o}$. Note that $x, x_{\alpha} \in V$ implies that $\operatorname{supp}\left(w_{x_{\alpha}}\right), \operatorname{supp}\left(w_{x}\right) \subseteq \bar{A}$. Therefore,

$$
\begin{aligned}
\left|w_{x_{\alpha}}(f)-w_{x}(f)\right| & =\left|\int_{Y} f d w_{x_{\alpha}}-\int_{Y} f d w_{x}\right|=\left|\int_{\bar{A}} f d w_{x_{\alpha}}-\int_{\bar{A}} f d w_{x}\right| \\
& =\left|\int_{\bar{A}}(g f) d w_{x_{\alpha}}-\int_{\bar{A}}(g f) d w_{x}\right|=\left|\int_{Y}(g f) d w_{x_{\alpha}}-\int_{Y}(g f) d w_{x}\right| \\
& =\left|w_{x_{\alpha}}(g f)-w_{x}(g f)\right|<\epsilon .
\end{aligned}
$$

Lemma 3.2. Let $X$ and $Y$ be locally compact Hausdorff spaces. Let $x \mapsto w_{x}$ be a compactly bounded continuous map from $X \mapsto\left(M_{b}(Y), \tau_{c}\right)$ such that $k=$ $\sup \left\{\left\|w_{x}\right\|: x \in X\right\}<\infty$. For each $g \in C_{b}(Y)$ define $g^{\prime}(x)=\int_{Y} g d w_{x}$ for all $x \in X$. Then

a) $g \in C_{b}(Y)$ implies $g^{\prime} \in C_{b}(X)$ and $\left\|g^{\prime}\right\| \leq k\|g\|$.

b) If $g_{\alpha} \rightarrow g$ in the strict topology and $\left\|g_{\alpha}\right\| \leq M<\infty \forall \alpha$, then $g_{\alpha}^{\prime} \rightarrow g^{\prime}$ in the strict topology.

Proof. a) Let $x_{\alpha} \rightarrow x$ be a net in $X$. Then, by Theorem 3.1, $w_{x_{\alpha}} \rightarrow w_{x}$ in the $C_{b}$-weak $\left(\tau_{b}\right)$ topology on $M_{b}(Y)$. Hence we have, for $g \in C_{b}(Y)$,

$$
g^{\prime}\left(x_{\alpha}\right)=w_{x_{\alpha}}(g) \rightarrow w_{x}(g)=g^{\prime}(x) .
$$

Thus $g^{\prime}$ is continuous. For the second part of a), note that

$$
\left|g^{\prime}(x)\right|=\left|w_{x}(g)\right| \leq\|g\|_{u}\left\|w_{x}\right\| \leq k\|g\|_{u} .
$$

b) Since $\left\|g_{\alpha}\right\| \leq M \forall \alpha$, we have that $\left\|g_{\alpha}^{\prime}\right\| \leq k M<\infty \forall \alpha$. Thus by Lemma 2.1, to show that $g_{\alpha}^{\prime} \rightarrow g^{\prime}(\beta)$ it is sufficient to show that $g_{\alpha}^{\prime} \rightarrow g^{\prime}(\kappa)$ (where $\kappa=$ the compact-open topology). Let $\epsilon>0$ be given and let $\phi \in C_{c}(X)$ (with $\|\phi\| \neq 0$ ). Then $X_{\phi}=\operatorname{supp}(\phi)$ is a compact subset of $X$. Since $w$ is compactly bounded, we have that $A=\bigcup_{x \in X_{\phi}} \operatorname{supp}\left(w_{x}\right)$ is a relatively compact subset of $Y$. Now, $g_{\alpha} \rightarrow g$ in the strict topology implies $g_{\alpha} \rightarrow g$ in the compact open topology which implies there is an $\alpha_{o}$ such that $\left\|g_{\alpha}-g\right\|_{\bar{A}}<\frac{\epsilon}{k\|\phi\|}$ for all $\alpha \geq \alpha_{o}$. Thus for $\alpha \geq \alpha_{o}$ we have

$$
\begin{aligned}
\left\|\phi g_{\alpha}^{\prime}-\phi g^{\prime}\right\| & =\left\|\phi\left(g_{\alpha}^{\prime}-g^{\prime}\right)\right\|=\sup _{x \in X}\left\{\left|\phi(x) \int_{Y}\left(g_{\alpha}-g\right) d w_{x}\right|\right\} \\
& =\sup _{x \in X_{\phi}}\left\{\left|\phi(x) \int_{Y}\left(g_{\alpha}-g\right) d w_{x}\right|\right\} \leq\|\phi\| \cdot k \cdot\left\|g_{\alpha}-g\right\|_{\bar{A}} \\
& <\|\phi\| \cdot k \cdot \frac{\epsilon}{k \cdot\|\phi\|}=\epsilon .
\end{aligned}
$$

Therefore $g_{\alpha}^{\prime} \rightarrow g^{\prime}$ in the strict topology. 
NOTE. From here on $M_{b}(X)$ is endowed with the $\tau_{b}$ topology unless otherwise noted.

Definition. Let $X, Y$ be locally compact. A map $T: M_{b}(X) \mapsto M_{b}(Y)$ is $X$ bounded if $\sup \left\{\left\|T\left(\delta_{x}\right)\right\|: x \in X\right\}<\infty$.

Theorem 3.3. Let $X, Y$ be locally compact. Let $x \mapsto w_{x}$ be as in Lemma 3.2. Then $\delta_{x} \mapsto w_{x}$ has a unique extension to an $X$-bounded, continuous map from $M_{b}(X) \mapsto M_{b}(Y)$.

[Please note that $D M_{b}(X)=\left\{\delta_{x}: x \in X\right\}$ is homeomorphic to $X$, and hence a continuous map from $X$ can be viewed as a continuous map from $D M_{b}(X)$.]

Proof. Let $\mu \in M_{b}(X)$. Then define $\phi(\mu) \in M_{b}(Y)$ via

$$
\phi(\mu)(g)=\int_{X} g^{\prime} d \mu \quad \forall g \in C_{b}(Y) \quad\left(\text { where } g^{\prime}(x)=\int_{Y} g d w_{x}\right) .
$$

We must verify that (1) $\phi(\mu) \in M_{b}(Y) \forall \mu$, (2) $\phi\left(\delta_{x}\right)=w_{x} \forall x \in X$ (which implies $\phi$ is $X$-bounded), (3) $\phi$ is linear and continuous, and (4) $\phi$ is unique.

For (1) we have that $M_{b}(X)=$ all linear functionals on $C_{b}(X)$ that are $\beta$ continuous on $\beta$-bounded sets (see p. 100 of [1]). Thus $\phi(\mu) \in M_{b}(Y)$ if and only if $\phi(\mu)\left(g_{\alpha}\right) \rightarrow \phi(\mu)(g)$ whenever $g_{\alpha} \rightarrow g$ in the strict topology and $\left(g_{\alpha}\right)$ is a $\beta$-bounded net in $C_{b}(Y)$ (equivalently a $\sigma$-bounded net). If $g_{\alpha} \rightarrow g$ is a $\beta$-bounded net, then, by Lemma 2.1, $g_{\alpha}^{\prime} \rightarrow g^{\prime}$ is a $\beta$-bounded net converging in the strict topology. Thus $\mu\left(g_{\alpha}^{\prime}\right) \rightarrow \mu\left(g^{\prime}\right)$ implies $\phi(\mu)\left(g_{\alpha}\right) \rightarrow \phi(\mu)(g)$ since $\phi(\mu)(f)=\mu\left(f^{\prime}\right)$ $\forall f \in C_{b}(Y)$.

For (2) note that $\forall x \in X$ and $g \in C_{b}(Y)$, we have that $\phi\left(\delta_{x}\right)(g)=\int_{X} g^{\prime} d \delta_{x}=$ $g^{\prime}(x)=\int_{Y} g d w_{x}=w_{x}(g)$. Thus $\phi\left(\delta_{x}\right)=w_{x}$.

For (3) $\phi$ is clearly linear and $\phi$ is continuous since if $\mu_{\alpha} \rightarrow \mu$ in $M_{b}(X)$, then $\mu_{\alpha}(f) \rightarrow \mu(f) \forall f \in C_{b}(X)$. Thus, since $f \in C_{b}(Y) \Rightarrow f^{\prime} \in C_{b}(X)$, we have that

$$
\phi\left(\mu_{\alpha}\right)(f)=\mu_{\alpha}\left(f^{\prime}\right) \rightarrow \mu\left(f^{\prime}\right)=\phi(\mu)(f) \quad \forall f \in C_{b}(Y) .
$$

For (4) let $\psi: M_{b}(X) \rightarrow M_{b}(Y)$ be any other continuous extension of $\delta_{x} \mapsto w_{x}$. Let $\mu \in F M_{b}(X)$. Then

$$
\mu=\sum_{n=1}^{m} \delta_{x_{n}} \Rightarrow \psi(\mu)=\sum_{n=1}^{m} \psi\left(\delta_{x_{n}}\right)=\sum_{n=1}^{m} w_{x_{n}}=\sum_{n=1}^{m} \phi\left(\delta_{x_{n}}\right)=\phi(\mu) .
$$

Thus $F M_{b}(X)$ dense in $M_{b}(X)$ implies $\psi=\phi$.

Lemma 3.4. Let $\mu \mapsto \mu^{\prime}$ be a continuous, linear and $X$-bounded map from $M_{b}(X)$ $\rightarrow M_{b}(Y)$. Also assume that for any compact subset $K \subseteq X$, the set $\bigcup\left\{\operatorname{supp}\left(\delta_{x}^{\prime}\right)\right.$ : $x \in K\}$ is relatively compact. For $g \in C_{b}(Y)$, define

$$
g^{\prime}(x)=\int_{Y} g d \delta_{x}^{\prime} \quad \forall x \in X
$$

Then,

a) $g^{\prime} \in C_{b}(X)$ and $\left\|g^{\prime}\right\| \leq k\|g\|$ where $\left.k=\sup \left\{\left\|\delta_{x}^{\prime}\right\|: x \in X\right\}<\infty\right]$, and

b) $\int_{Y} g d \mu^{\prime}=\int_{X} g^{\prime} d \mu$.

Proof. Since the map $x \mapsto \delta_{x}$ embeds $X$ into $M^{+}(X)$, we have that $x \mapsto \delta_{x}^{\prime}$ is continuous from $X \rightarrow M_{b}(Y)$. Thus a) follows from Lemma 3.2. 
By Theorem 3.3, $\mu \mapsto \mu^{\prime}$ is the unique $X$-bounded continuous extension of $\delta_{x} \mapsto \delta_{x}^{\prime}$ onto all of $M_{b}(Y)$. By the construction in the proof of Theorem 3.3, we have that

which is b).

$$
\int_{Y} g d \mu^{\prime}=\mu^{\prime}(g)=\int_{X} g^{\prime} d \mu
$$

Lemma 3.5. Let $X, Y, Z$ be locally compact. Suppose that $(x, y) \mapsto \omega_{x, y}$ is a compactly bounded and continuous map from $X \times Y \rightarrow\left(M_{b}(Z), \tau_{c}\right)$, and that $k=\sup \left\{\left\|\omega_{x, y}\right\|:(x, y) \in X \times Y\right\}<\infty$. Then

a) $\delta_{x} \times \delta_{y} \mapsto \omega_{x, y}$ has a unique $X \times Y$-bounded, $\tau_{b}$-continuous linear extension from $M_{b}(X \times Y) \rightarrow M_{b}(Z)$, and

b) $\left(\delta_{x}, \delta_{y}\right) \mapsto \omega_{x, y}$ has a unique extension to a bilinear map from $M_{b}(X) \times$ $M_{b}(Y) \rightarrow M_{b}(Z)$ that is separately continuous.

Proof. a) This is just Theorem 3.3 with $X$ replaced by $X \times Y$ and $Y$ replaced by $Z$.

b) The map $\left(\delta_{x}, \delta_{y}\right) \mapsto \omega_{x, y}$ is a composition of the maps $\left(\delta_{x}, \delta_{y}\right) \mapsto \delta_{x} \times \delta_{y}$ and $\delta_{x} \times \delta_{y} \mapsto \omega_{x, y}$. The second map has a unique continuous extension as in part (a) of this lemma. The first map can be extended to $(\mu, \nu) \mapsto \mu \times \nu$. Thus it suffices to show that $(\mu, \nu) \mapsto \mu \times \nu$ is continuous in each variable. Let $\mu_{\alpha} \rightarrow \mu$ in $M_{b}(X)$ and $\nu \in M_{b}(Y)$. We must show that $\mu_{\alpha} \times \nu \rightarrow \mu \times \nu$ in $M_{b}(X \times Y)$. Let $f \in C_{b}(X \times Y)$ and define $f^{\prime}(x)=\int_{Y} f(x, y) d \nu(y)$. If $f^{\prime} \in C_{b}(X)$, then we will have

$$
\begin{aligned}
\mu_{\alpha} \times \nu(f) & =\int_{X \times Y} f(x, y) d\left(\mu_{\alpha} \times \nu\right)(x, y) \\
& =\int_{X} \int_{Y} f(x, y) d \nu(y) d \mu_{\alpha}(x)=\mu_{\alpha}\left(f^{\prime}\right) \rightarrow \mu\left(f^{\prime}\right)=\mu \times \nu(f) .
\end{aligned}
$$

Thus it remains to show that $f^{\prime} \in C_{b}(X)$. Without loss of generality we may assume that $f \not \equiv 0$ and $\nu \not \equiv 0$. Fix $x_{o} \in X$ and let $\epsilon>0$ be given. Let $A \subseteq X$ be a compact subset such that $|\nu|(Y \backslash A)<\frac{\epsilon}{4\|f\|}$. Since $f$ is jointly continuous, we have that for each $y \in A$ there exist neighborhoods $U_{y}$ of $x_{o}$ and $V_{y}$ of $y$ such that

$$
\left|f\left(x_{1}, z_{1}\right)-f\left(x_{2}, z_{2}\right)\right|<\frac{\epsilon}{2\|\nu\|}
$$

for all $x_{1}, x_{2} \in U_{y}$ and $z_{1}, z_{2} \in V_{y}$. The collection $\left(V_{y}\right)_{y \in A}$ is an open cover of the compact set $A$; thus there is a finite subcover $\left(V_{y_{j}}\right)_{j=1, \ldots, n}$ of $A$. Let $U=\bigcap_{j=1}^{n} U_{y_{j}}$, which is an open neighborhood of $x_{o}$. Then for every $x \in U$ and $y \in A$ we have $\left|f(x, y)-f\left(x_{o}, y\right)\right|<\frac{\epsilon}{2\|\nu\|}$. Thus for every $x \in U$ we have

$$
\begin{aligned}
\left|f^{\prime}(x)-f^{\prime}\left(x_{o}\right)\right| & =\left|\int_{Y} f(x, y) d \nu(y)-\int_{Y} f\left(x_{o}, y\right) d \nu(y)\right| \\
& \leq \int_{A}\left|f(x, y)-f\left(x_{o}, y\right)\right| d|\nu|(y)+\int_{Y \backslash A}\left|f(x, y)-f\left(x_{o}, y\right)\right| d|\nu|(y) \\
& \leq \frac{\epsilon}{2\|\nu\|}\|\nu\|+2\|f\| \frac{\epsilon}{4\|f\|}=\frac{\epsilon}{2}+\frac{\epsilon}{2}=\epsilon .
\end{aligned}
$$

Thus $f^{\prime}$ is continuous. To see that $f^{\prime}$ is bounded, let $x \in X$. Then

$$
\left|f^{\prime}(x)\right|=\left|\int_{Y} f(x, y) d \nu(y)\right| \leq \int_{Y}|f(x, y)| d|\nu|(y) \leq\|f\| \cdot\|\nu\| .
$$


Theorem 3.6. Let $X$ be locally compact. Let $(x, y) \mapsto \delta_{x} * \delta_{y}$ be a continuous map from $X \times X \rightarrow\left(M_{b}(X), \tau_{c}\right)$ such that

i) $k=\sup \left\{\left\|\delta_{x} * \delta_{y}\right\|:(x, y) \in X \times Y\right\}<\infty$.

ii) For any compact subset $K \subseteq X \times Y$, the set $\bigcup\left\{\operatorname{supp}\left(\delta_{x} * \delta_{y}\right):(x, y) \in K\right\}$ is relatively compact.

Then the map $*$ has a unique extension to a bilinear map from $M_{b}(X) \times M_{b}(X) \rightarrow$ $M_{b}(X)$ that is separately $\tau_{b}$-continuous.

Proof. Let $Y=Z=X$ and apply Lemma 3.5.

With Theorem 3.6 proven the goal of this paper has been reached. This convolution extension theorem will allow one to study convolutions of measures, whether they be positivity-preserving or not, in terms of point-mass measures. In particular, with specific examples one may define a convolution of measures by merely defining a convolution of point-masses which satisfies the hypotheses of Theorem 3.6. Also, when checking that a defined convolution of measures is continuous with respect to the $C_{b}$-weak topology, one need only check $C_{c}$-weak continuity with respect to point-mass measures.

Remark. One of the more useful hypergroup axioms is the requirement that the mapping $(x, y) \mapsto \operatorname{supp}\left(\delta_{x} * \delta_{y}\right)$ be continuous with respect to the Michael topology (see [3], p. 12). The idea of a compactly bounded map is a weakening of the concept of continuity with respect to the Michael topology. Indeed, if $w: X \rightarrow$ $M_{b}(Y)$ yields continuity with respect to the Michael topology, then $w$ is compactly bounded (this is a result of Theorem 2.5f. in [3]). Significantly, it is merely compact boundedness, not continuity with respect to the Michael topology, that is needed to extend the convolution of point-mass measures to a separately $C_{b}$-weakly continuous convolution on all of $M_{b}(X)$. In [8] axioms for signed hypergroups were given by Rösler that abandoned the Michael topology for the very good reason that there are no nontrivial hypergroups on $\Re$ and that "this is mainly due to the hypergroup axiom on support continuity of the convolution with respect to the Michael topology" (9], p. 292). Rösler's replacement was the following:

(3.7) For each $f \in C_{c}(X)$ and any compact subset $K \subseteq X$, the set

$$
\bigcup_{x \in K}\left(\operatorname{supp}\left(T^{x} f\right) \cup \operatorname{supp}\left(T_{x} f\right)\right)
$$

is relatively compact in $X$, where $T^{x} f: y \mapsto \delta_{x} * \delta_{y}(f)$ and $T_{x} f: y \mapsto \delta_{y} * \delta_{x}(f)$.

Condition (3.7) seems to be closely related to compact boundededness. The author of this paper does not know if there exist any convolution structures which satisfy condition (3.7) but are not compactly bounded. The Laguerre Convolutions described in [8], and the Bessel-Type signed hypergroups described in [9] are examples of convolution structures which are compactly bounded and satisfy condition (3.7), but do not yield Michael topology continuity. Additional examples, in particular, signed quotient hypergroups, can be found in [10].

In conclusion, it may be that compact boundedness is a better replacement for the Michael topology continuity than condition (3.7) in the axioms of a signed hypergroup. Because $C_{c}$-weak continuity would imply $C_{b}$-weak continuity, compact boundedness would allow the $\sigma$-compact requirement in 8 ] to be dropped. It would also enable one to construct objects such as products and projective limits of signed hypergroups more generally than would otherwise be possible. Finally, compact 
boundedness would retain many of the useful results given by Michael topology continuity, such as, for example, the properties that the convolution of two compact sets will be (relatively) compact, and also that the convolution of two measures with compact support will again be a measure with compact support.

\section{REFERENCES}

[1] R.C Buck, Bounded continuous functions on a locally compact space, Michigan Math. J. (5) (1958), 95-104. MR 21:4350

[2] E. Hewitt, K.A. Ross, Abstract Harmonic Analysis, Vol. 1, Springer-Verlag, New York, 1979. MR 81k:43001

[3] R.I. Jewett, Spaces with an abstract convolution of measures, Advances in Math. (18) (1975), 1-101. MR 52:14840

[4] E.A. Michael, Topologies on spaces of subsets, Trans. Amer. Math. Soc. (71) (1955), 151-182. MR 13:54f

[5] W. Rudin, Functional Analysis, Second edition, McGraw-Hill, New York, 1991. MR 92k:46001

[6] A. Parr, Signed Hypergroups, Ph.D. Thesis, University of Oregon (1997).

[7] J.S. Pym, Weakly separately continuous measure algebras, Math. Ann. (175) (1968), 207-219. MR 36:5715

[8] M. Rösler, Convolution algebras which are not necessarily positivity preserving, Contemp. Math. (183) (1995), 299-318. MR 96c:43006

[9] M. Rösler, Bessel-type signed hypergroups on $\Re$, Probability measures on groups and related structures, XI (Oberwolfach 1994) (1995), 292-304. MR 97j:43004

[10] M. Rösler, M. Voit, Partial Characters and Signed Quotient Hypergroups, Canadian J. Math. (51(1)) (1999), 96-116. MR 2000g:43008

[11] K.A. Ross, Signed hypergroups-a survey, Contemp. Math. (183) (1995), 319-329. MR 96c: 43007

Department of Mathematics, University of the Virgin Islands, St. Thomas, United States Virgin Islands

E-mail address: aparr@uvi.edu 